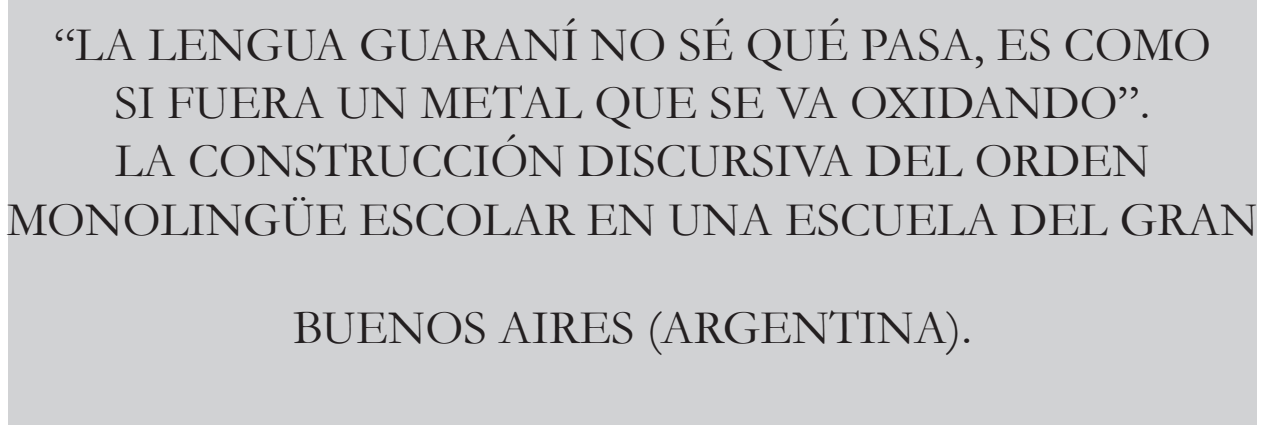

Cecilia Tallatta ${ }^{1}$

Virginia Unamuno ${ }^{2}$

Resumen: Este trabajo es parte de una etnografía realizada en una escuela del Gran Buenos Aires (Argentina). Niños y niñas provenientes de regiones plurilingües se escolarizan en una escuela que se reconoce a sí misma como monolingüe. Identificamos un conjunto de operaciones discursivas que participan en el "silenciamiento" de lenguas y que explican la perpetuación del "orden monolingüe escolar", así como estrategias de resistencia a dicho orden. Mostramos que, más que un orden dado, se trata de un proceso en el cual otras lenguas son "arrinconadas" hacia territorios simbólicos donde el entender (pero no hablar), lo doméstico y lo extranjero se conjugan para construir el territorio de "lo extraescolar". Esto permite poner en cuestión la idea del plurilingüismo escolar como la sumatoria de lenguas "presentes" en la escuela.

Palabras clave: Orden monolingüe escolar. Silenciamiento. Interacción. Resistencia.

\title{
“A LÍNGUA GUARANI, NÃO SEI O QUE ESTÁ ACONTECENDO, É COMO UM METAL QUE ENFERRUJA". A CONSTRUÇÃO DISCURSIVA DA ORDEM ESCOLAR MONOLÍNGUE EM UMA ESCOLA NA GRANDE BUENOS AIRES (ARGENTINA).
}

Resumo: Este trabalho faz parte de uma etnografia realizada em uma escola do Gran Buenos Aires (Argentina). Crianças que provém de regiões plurilingues são escolarizadas em uma escola que se reconhece como monolíngue. Identificamos um conjunto de operações discursivas que participam no "silenciamento" de línguas e que explicam a perpetuação da "ordem escolar monolíngue”, bem como estratégias de resistência a essa ordem. Mostramos que, mais do que uma ordem dada, trata-se de um processo em que outras línguas são "cercadas" em territórios simbólicos onde o entender (mas não falar), o doméstico e o estrangeiro se conjugam para construir o território do "extraescolar". Isso permite questionar a ideia do plurilinguismo escolar como a soma das línguas "presentes" na escola.

Palavras-chave: Ordem escolar monolíngue. Silenciamento. Interação. Resistência.

1 Doctora en Lingüística, Universidad de Buenos Aires. Becaria CELES/EH-UNSAM. E-mail: ceciliantl@gmail.com. 2 Doctora en Filología, Universitat de Barcelona. Investigadora CELES/CONICET. E-mail: vunamuno@conicet.gov.ar. 
“The Guarani language I don't know what happens, it's like it's a metal that rusts". The discursive construction of the monolingual school order in a school in Greater Buenos Aires (Argentina).

\begin{abstract}
This paper is part of an ethnography carried out in a school in Greater Buenos Aires (Argentina). Children from multilingual regions are enrolled in a school that identifies itself as monolingual. We identify a set of discursive operations that participate in the "silencing" of languages and explain the perpetuation of the "monolingual school order", as well as strategies of resistance to this order. We show that, rather than a given "order", it could be understood as a process in which other languages are "cornered" towards symbolic territories where "understanding (but not speaking)", domestic uses and foreign location are combined to build the "extracurricular" territory. This dynamic interpretation of "sociolinguistic order" allows us to question the idea of school multilingualism as the simple sum of languages "present" in the school.
\end{abstract}

Keywords: Monolingual school order. Silencing. Interaction. Resistance.

\section{Introducción}

Este artículo presenta los resultados de una etnografía sociolingüística crítica llevada a cabo en un centro escolar del conurbano bonaerense (Argentina), entre el 2014 y el 2018. El objetivo central fue relevar las formas en que se construye la “norma lingüística escolar", así como las prácticas de inclusión y exclusión que dicha norma realiza. Para el presente artículo, presentaremos un aspecto de esta investigación. Específicamente, abordaremos algunas de las características interaccionales del silenciamiento de las lenguas diferentes al castellano que consideramos resultan en el monolingüismo escolar. Nos enfocamos particularmente en algunos de los recursos interactivos que se despliegan en las aulas en relación con un orden monolingüe que sostiene ideologías lingüísticas que están en estrecha relación con ideologías nacionales.

Argentina es un país que se reconoce como monolingüe castellano. La escuela ha tenido históricamente - y aún lo tiene- un rol fundamental en la producción y reproducción de esta representación (BRIONES, 1998). Nuestro interés particular es desentrañar algunos de los recursos en los que esta producción-reproducción se basa.

Para ello, elegimos el caso de una escuela sita en el Gran Buenos Aires. Esta región está caracterizada por la presencia importante de personas provenientes de territorios bilingües o plurilingües, dentro y fuera de las fronteras estatales. Esta heterogeneidad lingüística es consecuencia de los diferentes movimientos migratorios que tuvieron lugar en las últimas décadas en el territorio argentino. Los datos del último Censo Nacional de Población, Hogares y Vivienda que se realizó en el año 2010 indican que el 60\% de la población no nativa del conurbano bonaerense proviene de países limítrofes, principalmente de Paraguay, Perú y Bolivia, cuyas constituciones los declaran como bilingües, en el primer caso, y plurilingües, en los últimos dos. A este porcentaje hay que sumarle el de la migración interna, dado que la mayor parte de los migrantes internos que habitan en esta región proviene de las provincias de Santiago del Estero y Corrientes (MARTÍNEZ, SPERANZA y FERNÁNDEZ, 2009). Ambas jurisdicciones se reconocen como bilingües: Santiago del Estero le asigna al quichua santiagueño el estatuto de "lengua de pervivencia" y Corrientes reconoce al guaraní como lengua co-oficial.

En este contexto, resulta necesario considerar que el plurilingüismo que observamos en el contexto en donde trabajamos -y en general en todo el territorio argentino- está signado por la desigualdad y el conflicto entre el castellano y las diferentes lenguas nativas, las que se convirtieron en "un estigma que generó un proceso de 
invisibilización de las mismas" (SPERANZA y PAGLIARO, 2015, p.11).

El caso que tomamos en el presente artículo es el de una escuela, "la Rivadavia"3. Su población escolar se caracteriza porque un $30 \%$ de alumnos poseen repertorios lingüísticos plurilingües. Estos repertoriosincluyen diversaslenguas sudamericanas: guaraní, aymara, quechua y quichua santiagueño. Se trata de niños y niñas que son miembros de familias migradas por razones económicas al partido de San Martín, que conforma el primer cinturón del Gran Buenos Aires.

Para este trabajo, nos centramos en el análisis de un conjunto de interacciones relevadas en el marco de un proyecto que buscó "poner en valor" la diversidad lingüística, así como las competencias de alumnos y alumnas en diferentes lenguas. En este marco, los hallazgos de nuestro estudio se hacen aún más importantes, porque muestran cómo aún en contextos de tematización explícita del plurilingüismo, el orden monolingüe escolar actúa "silenciando" las lenguas-otras.

Este artículo está organizado de la siguiente manera: en el segundo apartado, enmarcamos el problema a analizar dentro de las condiciones socio-histórico-políticas en las que se desarrolló la escuela estatal en Argentina y el rol que cumplió en el establecimiento y difusión del ideal del monolingüismo nacional. A su vez, vinculamos esta problemática con el estudio de las interacciones como una estrategia para el abordaje del orden sociolingüístico que organiza la participación en la Rivadavia. Luego, en el tercer apartado, describimos la población que conforma esta comunidad y la metodología desde la que abordamos el problema del silenciamiento de las lenguas-otras en el espacio escolar. A continuación, en el cuarto apartado, presentamos el análisis de una serie de fragmentos en los que se ponen en práctica

3 Todos los nombres que aparecen en el artículo, tanto de la escuela como de los participantes, son pseudónimos que utilizamos para proteger el anonimato de la investigación. distintos procedimientos discursivos que colaboran en el silenciamiento de las lenguas diferentes al castellano en las clases. Luego, en las conclusiones, recuperamos de manera esquemática los aportes de este estudio y, para finalizar, incluimos las referencias bibliográficas.

\section{Problema teórico y de investigación}

En Argentina, la escuela estatal es una de las instituciones que ha cumplido, desde su creación, un rol fundamental en la aplicación de las políticas lingüísticas que se han impulsado desde los diferentes gobiernos. En este sentido, tiene la función de regular la(s) lengua(s) oficial(es) y nacional(es) en el ámbito escolar. Su rol tradicional es, entre otros, el de colaborar en la reproducción de la hegemonía del castellano como lengua nacional y, de forma implícita o explícita según la ocasión, en la prohibición del uso de otras lenguas en el ámbito educativo, al menos. De este modo, el monolingüismo y la uniformidad social han dominado típicamente el sistema escolar al contribuir en la difusión del monolingüismo castellano como rasgo definitorio del ciudadano argentino (DI TULLIO, 2003; LÓPEZ GARCÍA, 2015; UNAMUNO, 1995).

La escuela estatal argentina comenzó a cumplir este rol con la promulgación, en el año 1884, de la Ley de Educación Común 1420 que estableció que la educación primaria tenía que ser común, laica, gratuita y obligatoria en todo el territorio nacional. Esta disposición formó parte de "la política de castellanización" que dominó el modelo de país de la generación del Ochenta, el cual se asentó en una “ideología romántica del monolingüismo estatal como constitutivo de una nación" (BEIN, 2010, p.309) y en una concepción de "ciudadano" que excluía a las comunidades indígenas que habitaban el territorio nacional. A su vez, con la gran inmigración de principios del siglo 
$\mathrm{XX}$, esta ley se consolidó como una herramienta de difusión de "la lengua y los valores del ser nacional" durante una etapa en la que ingresó al país gran cantidad de inmigrantes hablantes de otras lenguas, las que se sumaron a las lenguas preexistentes a la conquista de los pueblos originarios (BRIONES, 1998).

Desde el gobierno nacional se impulsó, entonces, la escolarización de todos los habitantes del país con el objetivo de que aprendieran la lengua y la cultura argentinas, y de que se conformara una identidad nacional que asimilara las múltiples colectividades con sus idiomas y tradiciones. De esta manera, la institución escolar colaboró en la difusión de la representación de los ciudadanos argentinos como hablantes monolingües de castellano y en la invisibilización de las otras lenguas que se habla(ba)n en el territorio argentino (GANDULFO 2007).

El ámbito legislativo, recién a comienzos del siglo XXI, reglamentó una ley nacional que incorpora la preocupación por la preservación de las lenguas y las culturas indígenas y de otras minorías. La reforma constitucional de 1994 incluyó por primera vez el derecho a una educación bilingüe e intercultural para las personas indígenas. A partir de dicha reforma, posteriormente, Ley de Educación Nacional 26.206 sancionada en el año 2006, instituye la Modalidad Intercultural Bilingüe (EIB) como dispositivo de política lingüística (UNAMUNO, 2015; UNAMUNO y BONNIN, 2018), reconociendo y valorando la multiculturalidad y el multilingüismo. De algún modo, por primera vez, el marco legal tensiona la producción simbólica de la "argentinidad" como monolingüe (UNAMUNO y MALDONADO, 2012).

Si bien se han analizado los discursos que acompañan la formación de las ideologías lingüísticas monolingües, menos énfasis se ha puesto en relevar los mecanismos a través de los cuales la construcción de este ideario se sostiene a lo largo del tiempo, a pesar de los cambios legistlativos y discursivos que se han venido produciendo. Entendemos que el estudio de las interacciones en las aulas puede aportar algunos elementos en este sentido. Particularmente, consideramos que permite observar mecanismos que participan en la circulación y reproducción de estas ideologías monolingües.

En el presente trabajo, nos interesa, así, desentrañar, a través del estudio de casos particulares, los modos en que la escuela argentina "silencia" otras lenguas para constituirse a sí misma en un espacio monolingüe. Según mostraremos, esto está vinculado a la instauración de un orden sociolingüístico que, como tal, controla y jerarquiza lo que en las instituciones pasa y se dice. Pero, como presentaremos, esto no implica necesariamente una aceptación sumisa de tal orden. La dialéctica entre reconocimiento y resistencia es el núcleo de nuestro trabajo.

La noción de orden sociolingüístico (MARTÍN ROJO et al., 2010) nos es útil para el análisis que realizamos. Por un lado, supone que existe una regulación de los usos lingüísticos que se realiza explícitamente, pero también implícitamente a través de las prácticas y de las formas en que dichos usos son categorizados en ellas. Por otro lado, la noción de orden sociolingüístico implica un cierto "régimen" (BLOMMAERT, 2010) que es reconocido por los participantes en las interacciones en que intervienen, orientándose hacia él o discutiéndolo.

Desde el punto de vista que queremos darle a este trabajo, el orden monolingüe escolar se describe como un conjunto de ideologías que operan en el campo del sentido común; es decir, se constituyen en los supuestos en que se apoya la co-construcción del sentido de las prácticas y de los enunciados. El estudio de las interacciones, en tanto que prácticas sociales y verbales, se propone 
así como una entrada posible a la descripción de dicho orden.

Concebimos a la interacción como un trabajo colaborativo de un hablante y un oyente que nos brinda una instancia elemental del orden social (GOODWIN, 1981). Así, el estudio de la interacción da elementos para entender el modo en que este orden se produce y se reproduce, al mismo tiempo que permite ver resistencias y puesta en juego de órdenes alternativos.

\section{Población y Método}

Este estudio se basa en el trabajo realizado en una escuela que, como se señaló, llamamos "la Rivadavia”. Durante varios años, Cecilia observó y registró las clases de primero y sexto grado. Además, llevó a cabo un proyecto colaborativo junto a las docentes Josefina e Isabella sobre las lenguas de la escuela, el cual culminó con el armado de un árbol genealógico-lingüístico por parte de los alumnos. La selección de estos dos grados del nivel primario resulta particularmente significativa por caracterizarse ambos como "lugares de pasaje" entre diferentes niveles educativos; $y$, a su vez, por presentar atributos diferenciados que se corresponden con los diferentes contextos en los que se ubican: primer grado es el extremo inicial del nivel primario y sexto grado, por su parte, es el escalafón final.

Sí bien tomamos en cuenta toda la etnografía como dato, en particular aquí nos enfocaremos en el análisis de la participación de dos estudiantes: Jonás y Alberto. Jonás es un alumno paraguayo de 7 años que cursa primer grado. Él se reconoce como hablante bilingüe guaraní-castellano y participa activamente en las actividades sobre la diversidad lingüística que realizamos en las clases. Sin embargo, en las tareas cotidianas de lectoescritura Jonás ocupa un rol secundario con pocas autoselecciones en las interacciones que estructuran las clases $y$, generalmente, cumplimenta con retraso los ejercicios que se le proponen. Por su parte, Alberto es un alumno paraguayo de 12 años que cursa sexto grado y que también se reconoce como hablante de guaraní y castellano. Él participa activamente en las clases autoseleccionándose, aunque encuentra dificultades para tomar el turno de habla, dado que su estilo participativo se distingue del de la comunidad de la Rivadavia -el que se caracteriza por presentar espacios de transición muy reducidos y solapamientos recurrentes- (TALLATTA, 2019). En el aula, Alberto cuenta anécdotas de sus viajes y expresa su vínculo fluido con Paraguay, nos expresa su felicidad cuando llega el verano porque pasa las vacaciones en la ciudad en la que nació, donde tiene parientes y amigos y se siente a gusto porque es libre para circular por las calles ya que conoce a todos.

La Rivadavia se sitúa dentro de Buenos Aires, provincia cabecera de Argentina. Se ubica, específicamente, en el partido de Gral. San Martín, uno de los 24 municipios que conforman el Conurbano Bonaerense y que se emplaza al noroeste de esta región. Este distrito cuenta con un $9,14 \%$ de población extranjera en total ${ }^{4}$; el porcentaje disminuye a menos de la mitad en el rango de edad de entre 5 y 14 años, que incluye, aproximadamente, los años de los alumnos que transitan la escolarización primaria.

A su vez, a partir de los datos censales, podemos identificar que los niños extranjeros de entre 0 y 14 años provienen mayormente de América (96,12\%). En especial de los siguientes países, ordenados de mayor a menor proporción: Paraguay (58,57 \%), Perú (21,84 \%) y Bolivia (8,71 $\%)$. Con respecto a la población total de niños de entre 0 y 14 años que habitan en el distrito, el porcentaje de niños paraguayos representa tan sólo

$4 \mathrm{El}$ porcentaje de población extranjera en los 24 partidos del Conurbano bonaerense representa el 7,49 \% de la población total. 
un $1,85 \%$, el de niños peruanos un $0,69 \%$ y el de niños bolivianos un $0,27 \%$.

Con respecto a la población que asiste a la Rivadavia, aproximadamente el $30 \%$ de los alumnos que asisten a esta institución tienen repertorios lingüísticos plurilingües que incluyen otras lenguas sudamericanas (guaraní, aymara, quechua y quichua santiagueño). Sin embargo, la escuela cuenta con pocos registros actualizados acerca del origen de la población. Los datos más actualizados son del año 2013, donde incorporan una columna que detalla "Oriundo de" (Argentina 77,10 \% - Paraguay 12,21 \% - Bolivia 2,29 \% - Perú 1,54 \% - Argentina (Paraguay) $)^{5}$ 3,05 \% - Argentina (Bolivia) 0,76\% Argentina (Perú) 0,76 \% - No registra 2,29\%).

Cuando ponemos en paralelo estos números con el porcentaje de niños de entre 0 y 14 años que el Censo Nacional del año 2010 contabilizó para el partido de Gral. San Martín, resulta significativa la proporción de alumnos extranjeros que asisten a la Rivadavia. Según los cálculos para el distrito entero, el porcentaje de niños paraguayos en edad de asistir a la escolaridad primaria representa tan sólo un 1,85\%, el de niños peruanos un 0,69 \% y el de niños bolivianos un 0,27 \%. En este sentido, la proporción que presenta esta institución supera en gran medida estos valores: los alumnos paraguayos representan un 12,21\% del total de la población de esta comunidad escolar, los alumnos bolivianos un $2,29 \%$ y los alumnos peruanos un $1,54 \%$. A su vez, los valores se distancian aún más cuando incluimos a los estudiantes que son argentinos pero tienen padres oriundos de otros países: 15,26\% oriundos de Paraguay; 3,05 \% oriundos de Bolivia y $2,30 \%$ oriundos de Perú.

En cuanto a la metodología, este trabajo se enmarca en una etnografía que se asocia a la propuesta que Rockwell (1985) denomina “estudios en casos" porque demanda la búsqueda

5 Las columnas indican entre paréntesis la nacionalidad de los padres cuando es distinta a la del hijo. de interpretaciones y de explicaciones en el exterior de las situaciones específicas que son objeto de estudio. En este sentido, el presente tiene que ser enfocado por el analista de manera tal de incorporar en su estudio las "huellas y contradicciones" que las dimensiones históricas dejan inevitablemente. De tal manera, entendemos a estas huellas y contradicciones como recursos para la resistencia que forman parte de la estructura compartida de la acción comunicativa (ROCKWELL, 2006).

Para el análisis de los datos, optamos por el enfoqueinteraccionalque consideraparticularmente el devenir de la acción conjunta. Mediante el estudio de los turnos de habla encontramos regularidades en las estrategias interaccionales utilizadas (GOODWIN, 1981). Las reparaciones son un recurso común al momento de poner en cuestión la competencia pasiva/activa en las lenguas-otras. A su vez, el estudio de la la indexicalidad de los pronombres espaciales nos permite describir los modos que interaccionalmente se establece un orden sociolingüístico que construye fronteras entre los espacios de uso de las diferentes lenguas involucradas. Un tercer recurso analítico consiste en la relativización de los saberes de los hablantes como estrategia para mantener a las lenguas-otras en el ámbito de lo privado dentro del aula.

En el próximo apartado, abordaremos también algunos aspectos de las prácticas de resistencia mediante la interrelación de los aspectos interaccionales y discursivos. Por su parte, estas acciones no responden a estrategias conscientes sino que están vinculadas a "complejos procesos cognitivos y sociales involucrados en la competencia comunicativa de cualquier hablante" (ROCKWELL, 2006, p.33). De este modo, las prácticas de resistencia que presentamos a continuación tienen en común el hecho de poner en duda la condición de verdad de lo dicho por la maestra. 


\section{Análisis}

Una de las maneras de entender la forma en que, como sostenemos, se produce localmente el monolingüismo escolar puede ser entrar en las aulas de la Rivadavia y escuchar las conversaciones que docentes y alumnos tienen allí. Para este trabajo, hemos seleccionado algunos fragmentos de clases en las cuales Jonás, alumno de primer grado, interactúa con sus compañeros y docentes. A través de Jonás, queremos dar voz a los jonases que en la Rivadavia hablan otras lenguas y aprenden a silenciarlas.

\section{Fragmento 1}

$\rightarrow 1$ JONÁS: cuando yo voy a paraguay $\uparrow$ (.) hablo $\downarrow$

2 JOSE: en qué hablás?

$\rightarrow 3$ JONÁS: en guaraní

$\rightarrow 4$ JOSE: ves:? el entiende guaraní

$5(2)$

6 CECI: muy bien $\downarrow$

7 (1)

8 JOSE: no era el [ver:de] ${ }^{1}$

9 DELFI: [en español] $]^{1}$ no [sé hablar ${ }^{2}$

10 CECI: $\quad[\mathrm{el} \text { verde } \downarrow]^{2}$ y sabés hablar en gua[raní? o podés $\uparrow$ eh 11 entender y no hablar $\downarrow]^{3}$

$\rightarrow 12$ JONÁS: [mirá $\downarrow$ (.) yo sé hablar en guaraní $]^{3}$ [lo hablo sólo $\uparrow().]^{4}$ sólo $\uparrow$ sólo en $\uparrow($.$) en$

13 JOSE:

$[(\text { inent. })]^{4}$

$\rightarrow 14$ JONÁS: el paraguay $\downarrow$

15 CECI: sólo en paraguay $\downarrow$ acá no $\downarrow$

16 JOSE: acá no:?

18 JOSE: no: $\uparrow$

$19(2)$

$\rightarrow 20$ JOSE: alguno más entiende el guaraní:?
Este fragmento está extraído de la actividad de "La flor de lenguas" "que busca relevar a través de un juego las lenguas que niños y niñas conocen o con las cuales tienen contacto. En la línea 1, Jonás responde a una pregunta que la docente hace en la clase "¿hay alguien que hable otra lengua?". Es interesante que su respuesta remite no a una lengua, sino a un lugar, poniendo énfasis en su experiencia subjetiva respecto del viaje que lo vincula con el lugar de origen de su familia. A continuación, manifiesta abiertamente hablar esta lengua (línea 3). Sin embargo, en la línea siguiente, su maestra, Josefina, reformula su respuesta y dice "él entiende". Por lo cual, indica al niño que escriba guaraní en el pétalo verde en el cual se enumeran las lenguas que uno "entiende pero no habla".

Cecilia repregunta a Jonás el alcance de su conocimiento sobre el guaraní para confirmar en el pétalo de qué color tenía que anotarlo (líneas 10 y 11). Él responde de manera anticipada y solapada que es hablante activo y competente en guaraní. Sin embargo, restringe su contexto de uso al territorio de Paraguay (líneas 12 y 14). A través de la identificación de este contexto de uso, Jonás describe interaccionalmente el vínculo entre las fronteras nacionales y las lenguas.

Según pudimos observar a lo largo del trabajo de campo, esta correspondencia entre los usos de las lenguas diferentes al castellano y los otros países, era común. Incluso resultaba habitual que los niños y las niñas se reconocieran como hablantes de "argentino", una categoría que se oponía a otras, como "boliviano" y "paraguayo".

Pero además, más allá de la correlación de diferentes hablas con diferentes estados, en las interacciones, las lenguas distintas al castellano eran asignadas al hogar, al espacio de lo privado, como puede verse en el siguiente fragmento:

6 Esta actividad fue una adaptación que realizó Cecilia junto a las maestras de una propuesta de trabajo que se encuentra en Virginia Unamuno (2012). Propuestas para la enseñanza de lenguas: lenguas en plural. Buenos Aires: Ministerio de Educación de la Nación 


\section{Fragmento 2}

1 CECI:y ustedes saben alguna otra:: $\uparrow$ saben cómo se dice sol o luna en alguna otra lengua?

2 JONÁS: [sí:]

$3 \mathrm{Av}^{7}: \quad[\mathrm{no:}:]$

$4(1)$

5 JOSE: en guaraní: $\uparrow$

$6(1)$

7 ALE: sí:! yo sé! yo sé! yo sé! yo sé!

8 JOSE: cómo se dice en guaraní?

9 ALE: eh:: no sé $\downarrow$

$10(2)$

$\rightarrow 11$ JOSE: pueden averiguarlo en casa eso eh $\downarrow$

12 CAR: jonás $\downarrow$

$13(3)$

14 CAR: jonás sabe $\downarrow$

$\rightarrow 15$ JOSE: pueden averiguarlo en ca:sa! (inent.) estas son?

16 CECI: vos sabés jonás? (.) cómo se dice sol?

18 CECI: cómo se dice?

19 JONÁS: (inent.)

20 CECI: [en guaraní sabés?]

21 ALE: [(inent.) fue a] aprende:r a hablar en inglés $\downarrow$

$22(3)$

23 JOSE: vamos así $\downarrow$

$24(1)$

25 JOSE: dos más $\downarrow$

$26(1)$

27 JONÁS: lo sé pero::^ no me lo acue:rdo $\downarrow$

28 CECI: [bueno $\downarrow$ ] no importa $\downarrow$ después cuando te acuerdes lo decís $\downarrow$

29 JOSE: [viste?]

En esta secuencia, luego de hacer una actividad que presenta una misma palabra en

7 La sigla "Av" indica que el turno lo emitieron varios alumnos de manera simultánea. cuatro idiomas — castellano, portugués, quichua e italiano-, Cecilia pregunta a los alumnos si alguno conoce cómo se dicen esos términos en otra lengua diferente a las mencionadas (línea 1). Jonás responde "sî", al mismo tiempo que los demás compañeros expresan al unísono que "no" (líneas 2 y 3). Su respuesta, entonces, queda superpuesta por las voces simultáneas del grupo.

A continuación, Josefina les plantea la posibilidad de averiguarlo en sus casas (línea 11). Frente a esta propuesta, Carlos se autoselecciona en la línea 2 para recuperar la respuesta de Jonás que no había tenido recepción. Expresa, entonces, que no es necesario esperar a volver a sus casas, porque su compañero lo sabe (líneas 12 y 14). Sin embargo, la maestra parece no escuchar la intervención de Carlos y continúa repitiendo que averigüen esas palabras, consultando a sus familiares (línea 15). Aunque en la clase anterior (ver Fragmento 1), Jonás se había reconocido como hablante activo de guaraní en Paraguay, en esta secuencia relativiza su competencia en esa lengua.

Lo que nos interesa destacar en este fragmento es que Jonás, finalmente, opta por alinearse a esta categorización (las lenguas que se hablan en casa, no en la escuela), y en la línea 27 manifiesta no recordar una palabra ("sol") que anteriormente había usado en guaraní frente a Cecilia.

Un último fragmento nos sirve para plantear una cuestión crucial en nuestro análisis: el modo en que los niños se alinean a las ideologías dominantes en la escuela y, al mismo tiempo, buscan responderlas.

En la clase siguiente, mientras realizan otra actividad, Jonás apela a Cecilia para recordarle que él sí sabe decir en otra lengua una de las palabras que están trabajando ("sol"). Nos interesa particularmente la intervención de Jonás en la línea 8. Ahí, pide a Cecilia que le cuente a la maestra (la "seño") que él conoce la palabra en guaraní y, 
en relevancia sus conocimientos sobre esta lengua. $\rightarrow 32$ JONÁS: qué me dijiste [de: la- ${ }^{5}$

\section{Fragmento 3}

$$
33 \text { CECI: } \quad[\text { lu:na } \downarrow]^{5}(.) \text { luna? }
$$

1 CAR: lo voy a hacer de vue:lta

2 CECI: [sí:? bueno $\downarrow]^{1}$

$\rightarrow 3$ JONÁS: [yo sé hablar] $]^{1}$ de so: $1 \downarrow$ (.) no te:: la acordás que te dije $\downarrow$

4 CECI: si::!

5 JONÁS: de so:1

$6(1)$

7 CECI: sí [(inent.) $]^{2}$

$\rightarrow 8$ JONÁS: [contale] ${ }^{2}$ a la se:ño $\downarrow$

9 CECI: y: luna también la sa- luna la sabés también?

34 JOSE: eso es ser (inent.)?

$\rightarrow 35$ JONÁS: ${ }^{\circ}(\text { jasy })^{\circ}$

36 CECI: ah::! Mirá $\downarrow$ [(.) qué $]^{6}$ li:ndo!

$\rightarrow 37$ JONÁS: $\quad[\mathrm{se}:-]^{6}$

$\rightarrow 38$ sé todo: yo!

39 CECI: todo: sabés todas las palabras?

40 CAR: no lo sabe todo!

41 CECI: no?

42 JONÁS: las pala:bras! Lo que dice está ahí $\downarrow$ (.) del á:rbol $\downarrow$

$43(5)$

44 ALE: seño!

10 JONÁS: sí

11 CECI: cómo es? Luna?

$\rightarrow 45$ CECI: ${ }^{\circ}$ (inent.) en el oído $\downarrow^{\circ}(($ me acerco a Josefina y le cuento))

$\rightarrow 12$ JONÁS: no me la acue:rdo $\downarrow$

46 JOSE: ((risas)) (inent.)

$\rightarrow 47$ JONÁS: le conta:ste?

14 JOSE: acá?

48 (1)

15 VIC: no: $\downarrow$ ahí es mejor hacer el á:rbol $\downarrow$

49 CECI: pero yo no las sé repetir $\downarrow$ no me sale $\downarrow$

16 JOSE: eh:: sí $\downarrow$ [(inent.) y te lo $]^{3}$ pego $\downarrow$

$\rightarrow 17$ JONÁS: $\quad$ [ahora sí me lo acue:rdo $\downarrow]^{3}[($.) mi $]^{4}$ rá $\downarrow$

$\rightarrow 51$ JONÁS: sé yo $\downarrow$

18

CECI:

[cómo

es? $]^{4}$

$\rightarrow 53$ JONÁS: le digo?

19 ALE: voy a escri:bi:r!

54 CECI: cómo es sol?

55 VALEN: queda alguna se:?

56 JONÁS: eh:?

$21(2)$

57 JOSE: (inent.)

58 CECI: sol $\downarrow$ cómo es?

59 (1)

60 JONÁS: eh:: $\downarrow$

$\rightarrow 24$ JONÁS: esperá que le hago al solci:to $\downarrow$

25 JOSE: dale $\downarrow$ (inent.)

26 CECI: una cari:ta $\downarrow$

28 ALE: este quién lo hizo?

$\rightarrow 29$ JONÁS: vení $\downarrow$

30 JOSE: za:ra: $\downarrow$

En el extracto anterior, se desarrolla una secuencia similar a la del fragmento 2 , en el que Jonás parece dudar respecto a su "habilitación" para hablar en guaraní en el aula. Lo hace alternando entre el "recordar" y "no recordar" poder hablar el guaraní (líneas 12, 17, 20 y 24). Finalmente, decide 
mantener su lengua en el espacio de lo privado, hablando a Cecilia al oído (línea 29). El siguiente fragmento de las notas de Cecilia después de la clase, explicitan este lugar de la investigadora como mediadora:

Notas de Cecilia (17/04/2015)

\begin{abstract}
"El silencio de esa lengua era tal que él no se atrevía siquiera a mencionar su nombre. ¿Será por ese motivo que Jonás me ubicó como mensajera entre él y Josefina? Yo acepté este rol y me acerqué a la maestra a contarle que Jonás me había hablado al oído en guaraní. Josefina se sorprendió de esta acción y sonrió valorando su estrategia. Sin embargo, no intervino recuperando este saber experto que le conté del alumno. Las secuencias quedaron como una anécdota de color que hablaban acerca de su timidez para hablar en público en guaraní. Esta ausencia de la puesta en valor del bilingüismo de Jonás me dio una pista para entender el pedido de intervención que él me había hecho y su consulta acerca de si estaba autorizado para hablarle en guaraní a Josefina. En este caso, incluso yo evité darle una respuesta habilitante a su pregunta porque reconocí que en la clase no se le estaba dando lugar a su competencia en otra lengua. Como compensación, intenté ponerla en valor mostrando interés y reconociendo su saber en tanto conocimiento al que sólo él tenía acceso".
\end{abstract}

En suma: según analizamos en las clases a través de las interacciones, las lenguas diferentes al castellano eran silenciadas en el ámbito escolar a través de tres procedimientos discursivos concurrentes: a. la localización de las lenguas fuera de las fronteras nacionales; b. la limitación de los saberes a las competencias lingüísticas pasivas (entender, pero no hablar); c. la marginación de los usos de estas lenguas al ámbito doméstico-familiar, extra-escolar. Nuestro argumento es que estas formas de categorizar las lenguas-otras refuerzan las fronteras simbólicas entre la(s) lengua(s) y sus espacios de uso habilitados, los cuales se sitúan fuera del ámbito escolar, describiendo en definitiva el ámbito escolar como monolingüe.

Sin embargo, como hemos señalado, estos procesos que denominamos "silenciamiento de las lenguas" no dejan de ser resistidos. Los niños y las niñas, al mismo tiempo que reconocen este orden monolingüe de las aulas y la escuela, ponen en juego estrategias para "dar voz" a las lenguas-otras.

A lo largo de la escolaridad, según hemos documentado, estas voces que hablan otras lenguas van silenciándose. Poco a poco, en las escuelas como la Rivadavia, las lenguas-otras se van arrinconando en los espacios domésticos, en esos otros países a donde, de tanto en tanto, uno viaja; en las voces de otras personas. Los niños van reconociendo el orden monolingüe de la escuela argentina y van arrumbando las lenguas-otras, guardándolas en esos lugares "íntimos". Pero ¿qué pasa con la lengua en estos procesos?

Para cerrar este apartado, nos gustaría dar voz a Alberto. Como Jonás, habla guaraní. Pero, a diferencia de Jonás, ya ha atravesado la escolaridad y está a punto de terminar la etapa primaria. En sexto grado, Jonás siente que su lengua guaraní “es un metal que se va oxidando".

\section{Fragmento 4}

\section{ALBER: una:}

2 ISA: sí ale

$\rightarrow 3$ ALBER: la lengua guaraní no sé qué pasa es como si fuera un metal que se va::

$\rightarrow 4$ oxidando (.) se va: pudrien:do como el metal y:: (.) si no se usa se destruye

5 LOREN: no se usa más

6 ALBER: ya no se usa más

8 ISA: ajá $\uparrow$

9 CECI: y cómo se pero por qué se pudre?

10 ISA: claro

11 CECI: vos pen-

$\rightarrow 12$ ALBER: por lo oxidado

$13(1)$

14 CECI: y la lengua cómo se pudriría?

$\rightarrow$ 16 ALBER: con las [(inent.) $]^{1}$ cada vez hablando (.) menos menos $[\text { menos }]^{2}$ 
17 ERI: [con el tiempo $]^{1}$

18 CECI:

$[\mathrm{ah}:: \text { : cada }]^{2}$ vez

19 hablando menos $\uparrow$

$\rightarrow 20$ ALBER: y hablando otro idio:ma

21 CECI: y cambiándola por otra

Este fragmento tuvo lugar en el marco de una serie de actividades que, como ya hemos mencionado, se orientaron a poner en valor la diversidad lingüística de los alumnos de la escuela. En este caso, se trata de una actividad que Cecilia llevó adelante en sexto grado. En particular, el extracto anterior formó parte de la puesta en común de una guía de preguntas que buscaba recuperar ideas y representaciones de los alumnos acerca de las lenguas ${ }^{8}$. Este fragmento presenta la respuesta de Alberto frente a la pregunta de “¿Cuántas lenguas creen que se hablan en el mundo?”. De este modo, Alberto transmite al grupo la experiencia de un hablante que siente la pérdida de su lengua: el guaraní. En este relato, él establece una analogía entre un metal oxidado y una lengua cuando se deja de hablar y se reemplaza por otra (líneas 4, 16 y 20). Cabe considerar que Alberto transitó todo el sistema educativo en Argentina, ya que se trasladó al país cuando tenía 4 años de edad. Creemos que la metáfora que emplea para hablar de su lengua resulta muy significativa para recuperar su perspectiva sobre los procesos sociolingüísticos de los que él es parte. De esta manera, el extracto anterior resulta particularmente significativo: hablar de esta pérdida en el espacio de las aulas en donde las lenguas-otras se silencian, niegan o marginan, es

8 1. ¿Qué creen que son las lenguas? / 2. ¿Cómo creen que se aprenden las lenguas? / 3. ¿Qué personas que ustedes conocen hablan lenguas diferentes? ¿Cómo se llaman estas lenguas? / 4. ¿Qué lenguas les parecen más importantes (de aprender, de saber, de hablar...)? ¿Por qué? / 5. ¿Conocen algún país en donde se habla una lengua diferente a la que ustedes hablan? ¿Cuál es? / 6. ¿Cuántas lenguas creen que se hablan en el mundo? ¿Y en la Argentina? ¿Y en nuestra ciudad? ¿Y en nuestra familia? - Este cuestionario es parte de una actividad para explorar las primeras impresiones de los alumnos acerca de las lenguas. Se encuentra completo en UNAMUNO, V. Propuestas para la enseñanza de lenguas: lenguas en plural. Buenos Aires: Ministerio de Educación de la Nación, 2012. tomar una posición importante, que puede leerse como un acto contestatario, un modo de resistencia imbuido de cierta nostalgia.

\section{Conclusiones}

En este artículo estudiamos el modo en que el orden social monolingüe atraviesa las prácticas discursivas e interaccionales en una escuela primaria estatal del conurbano bonaerense. Para ello, resultó indispensable incorporar en el análisis las coordenadas socio-histórico-políticas de la formación de las instituciones escolares y el rol que continúan cumpliendo en el establecimiento y la reproducción del ideal del monolingüismo nacional.

Mediante una metodología etnográfica nos acercamos a la Rivadavia y allí nos encontramos con discursos y prácticas interaccionales que se encuentran estrechamente ligados a ese ideal del monolingüismo castellano. Sin embargo, a través de un enfoque interaccional de los datos, nos fue posible identificar determinadas huellas y contradicciones como recursos para la resistencia frente al silenciamiento de las lenguas-otras en el espacio escolar.

En este sentido, identificamos tres procedimientos discursivos complementarios que colaboran en la reproducción del monolingüismo en las clases: a. la localización de las lenguas diferentes al castellano fuera de las fronteras nacionales; b. la limitación de los saberes de los alumnos a las competencias lingüísticas pasivas (entender, pero no hablar); y c. la marginación de los usos de estas lenguas al espacio doméstico-familiar, extra-escolar. Sin embargo, este análisis interaccional de los datos nos permitió también identificar algunas estrategias de resistencia por parte de los alumnos que disputan la condición de verdad del monolingüismo. Las reparaciones, la indexicalidad de los pronombres espaciales, la relativización de las competencias en 
las lenguas-otras y el intento de que esas lenguas participen de las clases son estrategias de resistencia que, a primera vista, resultan contradictorias pero que comparten el rol de disputar las fronteras que establecen determinados espacios de uso para la(s) lengua(s).

Nuestro análisis propone entender el "monolingüismo escolar" como un proceso de legitimación de un orden sociolingüístico particular que opera a través de la interacción y, en ella, de la categorización de los recursos y usos verbales de los participantes. Esto nos lleva a considerar que el plurilingüismo escolar o la diversidad lingüística en las aulas no resulta de la simple presencia de hablantes de otras lenguas en ellas. Contrariamente, como hemos mostrado, estas lenguas pueden acallarse para perpetuar un orden monolingüe aún en contextos en donde otras lenguas están presentes.

En el caso que analizamos, incluso en el marco de una propuesta didáctica que tematiza explícitamente el valor de la diversidad lingüística, se producen situaciones que evocan este orden y que muestran aspectos de su funcionamiento regulador.

Resultados parecidos a los que presentamos fueron expuestos por Martín Rojo y su equipo (MARTÍN ROJO, 2010). En su trabajo en aulas de secundario en Madrid, ella muestra diversos recursos a través de los cuales se construye el orden sociolingüístico monolingüe escolar, así como sus consecuencias en la vida académica y social de los jóvenes.

Sin embargo, a diferencia de Martín Rojo (2010), nuestro estudio no focaliza los modos en que se impone la hegemonía del español (y de una determinada variedad de esta lengua) a través de la interacción, sino en los modos en que las otras lenguas van "sacándose" del aula hacia otros confines, en una dinámica que distingue entre lo escolar/no escolar, que resultarían de la materialización de las fronteras entre el espacio de lo público y lo privado.

Resta aún profundizar en el estudio de las estrategias de resistencia como prácticas que colaboran en la distribución de las voces y las lenguas en las clases. Futuros estudios podrían abordar la influencia que estas estrategias tienen en el discurso docente y en la relativización de las afirmaciones de las maestras. Incluso resultaría particularmente interesante el análisis de las estrategias de resistencia de las y los docentes frente a la reproducción del orden sociolingüístico?.

\section{Referencias}

BEIN, R. Los meandros de la política lingüística argentina en relación con las lenguas extranjeras en las leyes generales de educación. En: ARNOUX, E. y BEIN, R. (comps.). La regulación política de las prácticas lingüísticas. Buenos Aires: Eudeba, 2010.

BLOMMAERT, J. The sociolinguistics of globalization. Cambridge, UK; New York: Cambridge University, 2010.

BRIONES, C. La alteridad del "Cuarto Mundo". Una deconstrucción antropológica de la diferencia. Buenos Aires: Ediciones del Sol, 1998.

DI TUlLIO, Á. Políticas lingüísticas e inmigración. El caso argentino. Buenos Aires: Eudeba, 2003.

GOODWIN, C. Conversational Organization. Interaction between speakers and hearers. Nueva York: Academic Press, 1981.

LÓPEZ GARCÍA, M. Nosotros, vosotros,

9 CRITERIOS DE TRANSCRIPCIÓN: [solapamiento] solapamiento entre dos intervenciones/ $\uparrow$ entonación ascendente / $\downarrow$ entonación descendente / ? pregunta / (.) pausa breve de menos de 1 segundo / (2) pausas cronometradas en segundos / : alargamiento vocálico / - paro repentino, interrupción / (inent.) fragmento inaudible / (duda) hipótesis del fragmento / ((risas)) anotación de actividad no verbal / ovolumen ${ }^{\circ}$ disminución del volumen de la voz 
ellos. La variedad rioplatense en los manuales escolares. Ciudad de Buenos Aires: Miño y Dávila editores, 2015.

MARTÍN ROJO, L. Constructing inequality in multilingual classrooms. Berlín: Mouton de Gruyter, 2010.

MARTÍNEZ, A. (coord.); SPERANZA, A. y FERNÁNDEZ, G. El entramado de los lenguajes. Una propuesta para la enseñanza de la Lengua en contextos de diversidad cultural. Buenos Aires: La Crujía, 2009.

ROCKWELL, E. Etnografía y teoría de la investigación educativa. En: Dialogando, v. 8, pp. 1-18, 1985.

ROCKWELL, E. Resistencia en el aula: entre el fracaso y la indignación. En: Educação em Revista, Belo Horizonte, v. 44, pp. 13-39, dez. 2006.

SPERANZA, A. y PAGLIARO, M. Reflexiones sobre la diversidad lingüística y cultural en el conurbano bonaerense. En: Revista de Políticas Sociales. Invierno 2015, 1: 2, pp. 11-15, 2015.

TALLATTA, C. ¿iQue hable fuerte!, ¿Qué? ¿tenés miedo?’ Interacción, normatividad y políticas del lenguaje en una escuela con población migrante. Facultad de Filosofía y Letras, UBA. Tesis doctoral, 2019.

UNAMUNO, V. Hacia una descripción del proceso de sustitución de la lengua guaraní en un barrio marginal del Gran Buenos Aires. Algunas consideraciones. En: Actas II Jornadas de Lengua Aborigen (pp. 469-477). Buenos Aires, Instituto de Lingüística, Universidad de Buenos Aires, 1995.

UNAMUNO, V. Propuestas para la enseñanza de lenguas: lenguas en plural. Buenos Aires: Ministerio de Educación de la Nación, 2012.

UNAMUNO, V. Los Hacedores de la EIB: Un Acercamiento a las Políticas LingüísticoEducativas Desde las aulas Bilingües del Chaco. En: Education Policy Analysis Archives, 23, pp. 1-35, 2015.
UNAMUNO, V. y BONNIN, J. E. "We Work as Bilinguals": Socioeconomic Changes and Language Policy for Indigenous Languages in El Impenetrable. En: TOLLEFSON J. W. and PÉREZ-MILANS, M. (Eds.), The Oxford Handbook of Language Policy and Planning (379397). Oxford: Oxford University Press, 2018.

UNAMUNO, V. y MALDONADO, Á. Prácticas y repertorios plurilingües en Argentina. Bellaterra: GREIP, 2012.

Submissão: outubro de 2019. Aceite: janeiro de 2020. 